\title{
Central-Bank Independence, E conomic Behavior, and Optimal Term Lengths: Comment
}

\author{
Xiang $\operatorname{Lin}^{\infty}$ \\ Department of E conomics, Stockholm University \\ First version: September 1997
}

\begin{abstract}
A bstract
Waller and Walsh (1996) argue that the optimal term length of the central banker can exceed one period when the central bank is conservative enough. However, the optimal conservativeness is unlikely to be exogenous. In this note we show how the optimal conservativeness and the optimal term length are determined simultaneously in the framework of Waller and Walsh. Furthermore, we extend the study to the $\mathrm{in}^{\circ}$ ation contract and the $\mathrm{in}^{\circ}$ ation target regimes. Under both regimes, the optimal parameter of conservativeness is independent of the term length and is always 1. Moreover, it is possible of have an optimal multi-term central banker under both the state-contingent in ${ }^{\circ}$ ation contract regime and the state-contingent in ${ }^{\circ}$ ation target regime.
\end{abstract}

Keywords: central bank independence, in ${ }^{\circ}$ ation target, and optimal term length. J EL Classi ${ }^{-}$cation Number: E52, E58.

$\mathrm{I}$ would like to thank Mats Persson for his discussions and suggestions. M ail address: Department of Economics, Stockholm University, S-106 91 Stockholm, Sweden. E-mail: xl@ne.su.se 
In an article recently published in the American Economic Review, Waller and Walsh (1996) (referred to henceforth as W \&W) o optimal term length and the independence (conservativeness) of the central bank. They conclude that when there is political uncertainty and persistent shifts in public preferences, the appointment of a conservative central banker is able to increase the optimal term length and therefore lead to lower average in ${ }^{\circ}$ ation, without necessarily increasing the volatility of output. The (necessary) condition for achieving the optimal multi-term length is that the parameter of conservativeness should exceed a constant critical value. They treat the parameter of the conservativeness as a given constant throughout their paper. However, as suggested by many other studies, the degree of the conservativeness should be optimally determined. By introducing a determination of the optimal parameter of the conservativeness into the $W \& W$ model, we obtain a sequence of optimal parameters associated with the term length instead of a single constant. Furthermore, from our point of view, the optimal term length and the optimal conservativeness should be determined simultaneously. Therefore, the longest optimal term length might not be the optimal solution for welfare maximization.

In this note, we show how the optimal conservativeness and the optimal term length are determined simultaneously on the basis of the $W \& W$ model. We also give an example to demonstrate the possibility of a multi-equilibrium solution. Furthermore, we study the case of $a^{\circ} i^{\circ}$ ation contract and an in ${ }^{\circ}$ ation target, which are mentioned by $\mathrm{W} \& \mathrm{~W}$ without any clear conclusion. We conclude that the parameter of optimal conservativeness is always 1 under both the in ${ }^{\circ}$ ation contract regime and the in ${ }^{\circ}$ ation target regime. Under both the constant in ${ }^{\circ}$ ation contract and the constant in ${ }^{\circ}$ ation target regimes, the optimal term length is always oneterm. Moreover, it is possible to have an optimal multiterm central banker under the state-contingent in ${ }^{\circ}$ ation contract and the state-contingent in ${ }^{\circ}$ ation target regimes.

\section{Simpli ${ }^{-}$ed W \&W Model and Solution}

We ${ }^{-}$rst simplify the model established by $W \& W$, since many factors in the original paper do not seem to be important for the issue of determining the optimal term length $\mathrm{T}$ and the optimal conservativeness ${ }^{-}$. Ignoring the money market (the velocity shock 
and the monetary shock) and the supply shocks speci ${ }^{-} \mathrm{c}$ to individual sectors would have no consequences for the discussion in this note. We here assume that the central bank is able to control in ${ }^{\circ}$ ation completely and precisely.

The (log) sectorial output $y_{i t}$ is determined as follows:

$$
y_{i t}=y^{N}+,\left(1 / 4 i^{1 / 4}\right)+u_{t} ;
$$

$(\log ) \mathrm{y}^{\mathrm{N}}$ is the natural rate of output and is a constant across the sectors. $1 / 4$ is the in $^{\circ}$ ation rate and $1 / 4$ is the expected in ${ }^{\circ}$ ation rate based on the information available at time $t$ i 1 : The economy-wide supply shock $u_{t}$ is a zero-mean process with a constant variance 3/4:

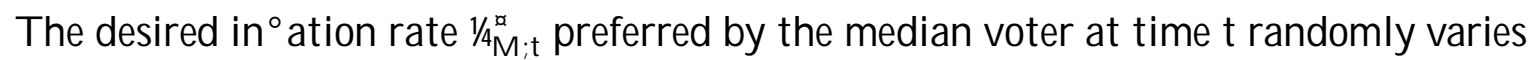
around the mean $\stackrel{\alpha}{\mathrm{M} ; \mathrm{t}}$ :

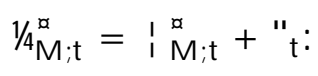

The mean $\stackrel{\infty}{M} ; t$ allows for persistent but infrequent shifts:

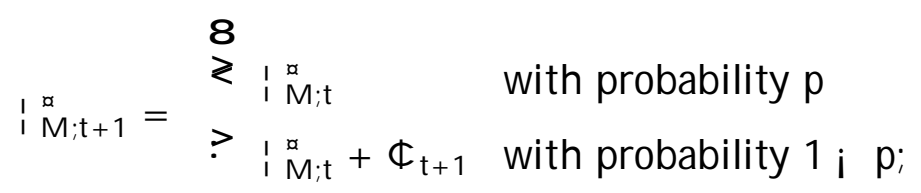

where $\phi$ is a zero-mean process with a constant variance $3 / 4.4$. The in ${ }^{\circ}$ ation rate preferred by the central bank depends on the degree to which it is subject to partisan interests:

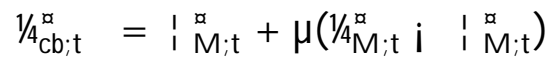

$$
\begin{aligned}
& =\mid \stackrel{\infty}{M ; t}+\mu^{\prime \prime}{ }_{t}:
\end{aligned}
$$

The parameter $\mu$; which is between 0 and 1 ; re ${ }^{\circ}$ ects the degree of partisanship in the appointment process. If $\mu=0$; the central banker merely follows the mean.

The loss functions for each individual sector are given by

$$
L_{i t}=\frac{1}{2}\left[\left(y_{i t} \text { i } y^{N} \text { i } k\right)^{2}+\mathbb{A}\left(1 / 4 ; \quad 1 / \frac{\alpha}{4}\right)^{2}\right] ;
$$

where $1 /{ }^{\alpha}{ }^{\alpha}$ is the $\mathrm{in}^{\circ}$ ation rate desired by sector $\mathrm{i}$ and is assumed to be distributed uniformly over the range $[\mathrm{L} ; U]$ with a constant variance $3 / 4 / 4$. The central bank has its own loss function:

$$
L_{t}^{C B}=\frac{1}{2}\left[\left(y_{t}^{C B} ; y^{N} ; k\right)^{2}+\circledR^{-}\left(1 / t^{C B} ; 1 / \frac{a}{t} b ; t\right){ }^{2}\right] ;
$$

where $^{-}, 1$; indicating that the central bank can be more conservative than the society. 
We suppose that the monetary policy is carried out by an instrument independent central bank. In other words, the central bank can carry out the monetary policy independently in order to minimize its own loss function (6). A party is able to in ${ }^{\circ}$ uence monetary policy via the appointment of a central banker with a certain degree of conservativeness ${ }^{-}$and term length $\mathrm{T}: \mathrm{A} n$ in $^{\circ}$ uence can also be exerted by other factors, such as the parameter $\mu$; as indicated in (3).

$\mathrm{The}^{\circ}{ }^{\circ}$ ation rate and the output in equilibrium under the objective function of the central bank (6) can be expressed by

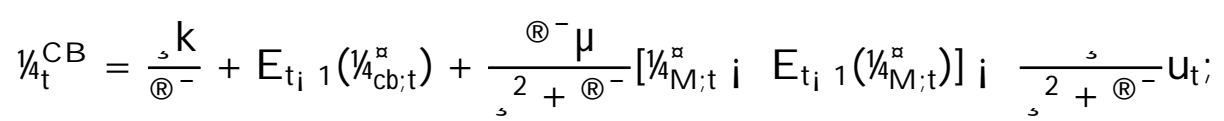

and

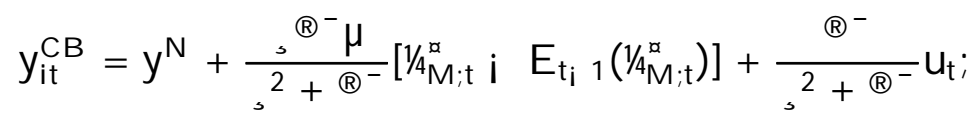

respectively. The socially expected average loss can be obtained by substituting (7) and (8) into (5). The average expected loss under a multi-term (T) central banker is then

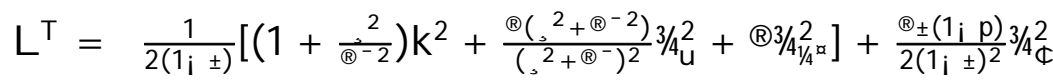

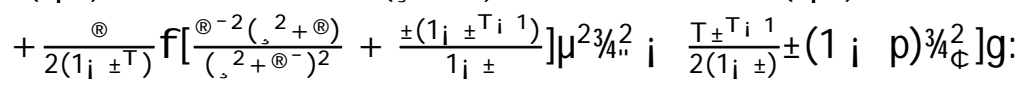

Following $W \& W$, we can ${ }^{-}$nd the relationship between the optimal term length $T$ and conservativeness $^{-}$:

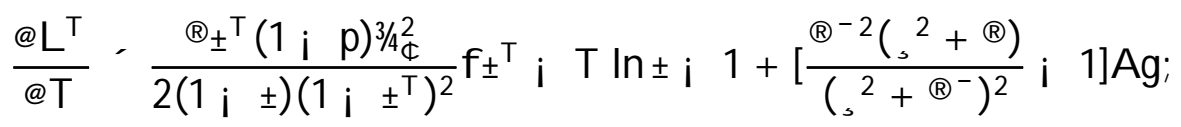

where $A^{\prime} \quad \frac{\left(1_{i} \pm \ln \pi^{23 / 4}\right.}{\left(1_{i}\right)^{3 / 4}}<0$ : W hen the conservativeness ${ }^{-}$is given, we can ${ }^{-}$nd out whether the optimal term length exceeds one period by using the necessary condition stated by W \&W . If ${ }^{-} \cdot \mathrm{H}$; where $\mathrm{H}=1+\left(1+{ }^{2}=\mathbb{B}\right)^{1=2}>1$; the optimal term length $\mathrm{T}$ should be one period. If ${ }^{-}>\mathrm{H}$; it is possible that $\mathrm{T}^{>}>1$ which ful $^{-}$Ils the following condition

$$
\pm^{\top} \mathrm{i} T \ln \pm, 1+\left[1 ; \frac{{ }^{-2}\left(,^{2}+\circledR\right)}{\left(,^{2}+\AA^{-}\right)^{2}}\right] \mathrm{A}:
$$

By ignoring the integer constraints of the ${ }^{-}$rst order condition, $\frac{\Phi^{\top}}{\Phi^{\top}}=0$, we can ${ }^{-}$nd the relationship between the optimal term length and the conservativeness:

$$
-=\frac{,{ }^{2} \mathrm{~B}}{1 \mathrm{i}{ }_{\mathrm{RB}}}
$$


where $B{ }^{\prime} \frac{{ }^{3} A+ \pm_{i} T \ln \pm 1}{\left.i A \Theta,{ }^{2}+\Theta\right)}{ }^{\frac{1}{2}}>0$. Figures 1 and 2 show the simulation result of the ${ }^{-}$rst order condition (the solid lines in Figure 1 and the dashed line in Figure 2). A $n$ increase of the conservativeness - would lead to an increase in the optimal term length $\mathrm{T}$. However, the optimal term length $\mathrm{T}$ can only approach its upper limit asymptotically (for instance $\mathrm{T}=23$ in Figure 1).

\section{Conservativeness}

W \&W treat the parameter - as a given constant. However, many other studies, for instance the studies of R ogo $®$ (1985) and A lesina and Gatti (1995), have suggested that the parameter of conservativeness - ${ }^{-}$should be optimally determined and therefore should be determined in conjunction with the optimal term length. We ${ }^{-}$rst consider the relationship between the optimal conservativeness and the term length. If the central bank in the study is completely partisan, the decision is based on the incumbent's objective function. Therefore we have to use the median voter's loss function as an indirect objective function to identify the optimal parameters. However, according to the setting by $W \& W$, the only di ßerence among the sectors is the desired rate of in ${ }^{\circ}$ ation $1 / 2 \alpha$; which has been treated as

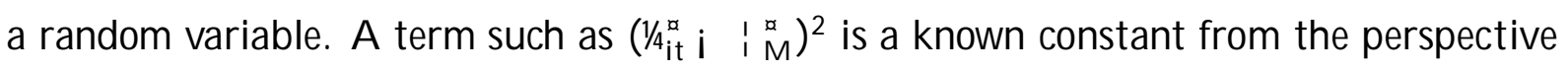
of an agent in sector $i$; its expectation from the perspective of sectorial anonymity is $3 / 4 / 4 / 4$ : Moreover, it will be clear later that $3 / 4 / 4$ has no power to in ${ }^{\circ}$ uence the decision on the optimal parameter ${ }^{-}$and the optimal term length T. Hence it is not important who is to appoint the central banker, since the optimal decision could be based on the average expected social loss function (9).

In the absence of political uncertainty, i.e., $3 / 4=3 / 4=0$; the average loss function is reduced to

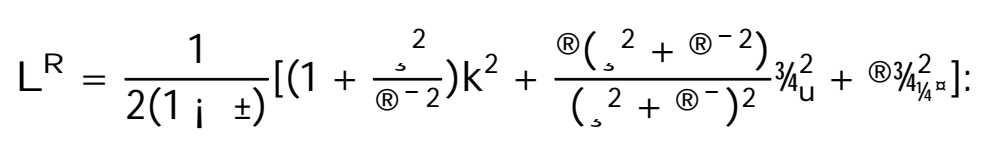

The ${ }^{-}$rst order condition,

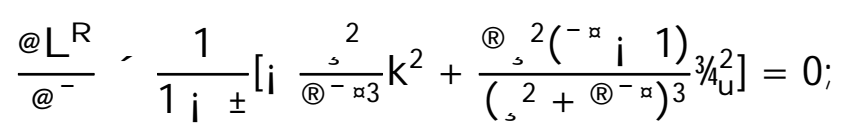

then indicates that the optimal parameter ${ }^{-}$has to be larger than 1: Thus, as shown by $\operatorname{Rogo}(1985)$, a more conservative central bank $\left(^{-\infty}>1\right)$ is an optimal choice. 
When political uncertainty is introduced into the model, the optimal conservativeness would rely on the size of the shocks. By considering the case that $T=1$; the ${ }^{-}$rst order condition,

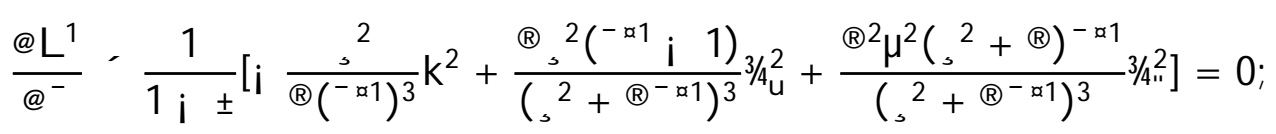

suggests that the optimal conservativeness ${ }^{-x 1}$ would be smaller than that in the absence of political uncertainty ${ }^{-}$: (Like most other studies, we here ignore the multi-solution.) An important fact is that a more conservative central bank may not be the optimal choice if the political uncertainty $3 / 4$ is large enough. Considering the constraint condition, - . 1; the optimal parameter ${ }^{-}{ }^{x 1}$ could reduce to $1:$ This is because within the framework proposed by $W \& W$, the political uncertainty makes no contribution to the level of the mean, or of the in ${ }^{\circ}$ ation bias. In other words, the in ${ }^{\circ}$ ation bias and the expected in ${ }^{\circ}$ ation rate would not be a ßected by the political uncertainty. The uncertainty has eßects merely on the second moment of the variables.

In the case of a multi-term central banker, the ${ }^{-}$rst order condition with respect to the parameter ${ }^{-}$indicates

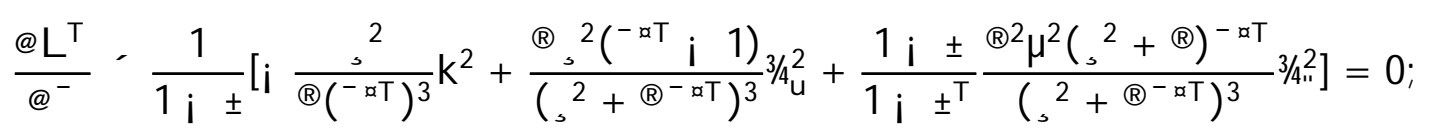

where $T, 1:$ Thus we have a sequence of optimal parameters of conservativeness $f^{-x T} g$ corresponding to the term length $\mathrm{T}$ : By neglecting the integer constraint, ${ }^{-\mathrm{xT}}$ may be regarded as a function of $\mathrm{T}$ : The simulation result in Figure 2 (the solid line) shows that ${ }^{-}{ }^{x T}$ increases monotonically corresponding to the increase of $T$ : This can be easily identi ${ }^{-}$ed by rewriting (12):

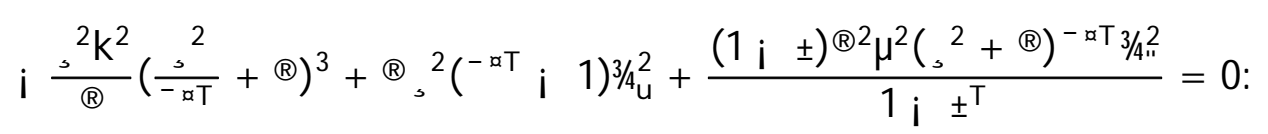

We then have

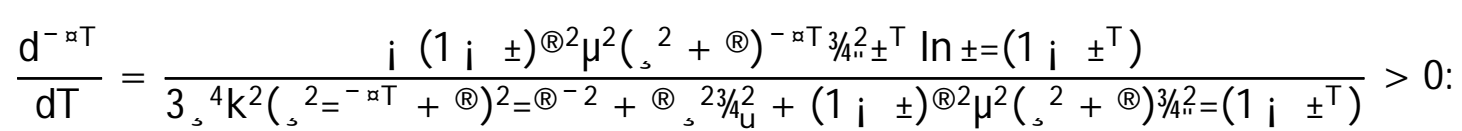

We can therefore conclude that ${ }^{-}>^{-\mathrm{x} T}>^{-{ }^{-} 1}$ : So an increase of the term length $T$ would lead to a higher optimal conservativeness ${ }^{-\mathrm{xT}}$.

When either $k$ is small or the shock $3 / 4$ is large enough, ${ }^{-}{ }^{x}$ could be smaller than $H$. However, it is also possible for the parameter ${ }^{-x}$ to exceed $H$ after some periods, as shown in Figure 2. 
If we put the two - rst order conditions (11) and (12) together, we can obtain the optimal conservativeness ${ }^{-}$and the optimal term length $\mathrm{T}$ : As indicated in Figure 1 , the optimal term length could reach 23 terms, but the optimal term length associated with the optimal conservativeness is approximately 4 terms (equilibrium II in Figure 2). State $\mathrm{I}$ in Figure 2 is not an equilibrium. However, for ${ }^{-x}<\mathrm{H}$ we have another equilibrium, that is, ${ }^{-x}$ and $T^{x}$ equal to 1 : So we have a multi-equilibrium solution.

\section{$3 \mathrm{In}^{\circ}$ ation Contracts and Optimal $\mathrm{In}^{\circ}$ ation Targets}

$\mathrm{W} \& W$ have mentioned that a study of the optimal term length with the in ${ }^{\circ}$ ation contract is of importance, but they have not given an explicit discussion. Furthermore, many countries have adopted in ${ }^{\circ}$ ation targets as a means to reduce in ${ }^{\circ}$ ation in reality, so it is worth carrying out a study of the target regime. In this note, we study the optimal term length associated with the in ${ }^{\circ}$ ation contract regime suggested by Walsh (1995) and Persson and Tabellini (1993) and the optimal term length associated with the ${ }^{\circ}{ }^{\circ}$ ation target regime suggested by Svensson (1997). Since the desired in ${ }^{\circ}$ ation rate is a random variable, the contract and the target could rely on the state of the realized shock ". Therefore, two cases are considered in our study, the constant in ${ }^{\circ}$ ation contract and target and the state-contingent in ${ }^{\circ}$ ation contract and target.

\subsection{In $^{\circ}$ ation Contracts}

We ${ }^{-}$rst consider the constant in ${ }^{\circ}$ ation contract. We suppose that the median voter can

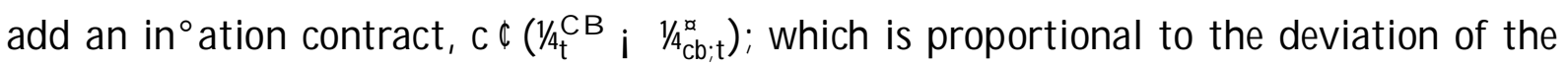
in ${ }^{\circ}$ ation rate from the desired level, to the central bank's loss function (6):

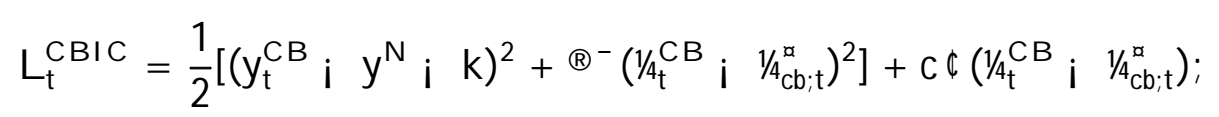

where $\mathrm{c}$ is a constant, that is, $\mathrm{c}=\mathrm{a}$. The economic intuition underlying (13) is that the in ${ }^{\circ}$ ation contract puts more weight on in ${ }^{\circ}$ ation stability. The ${ }^{-}$rst order condition for minimizing (13) is

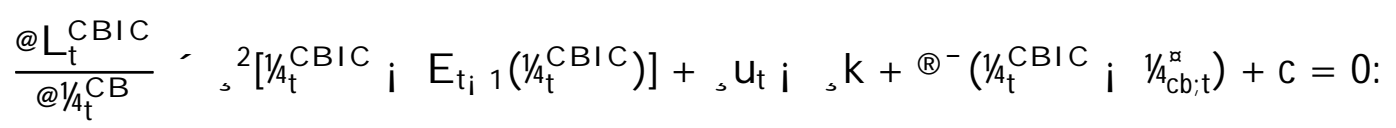


Taking the expectation on both sides of the ${ }^{-}$rst order condition (14) based on the information available at period $t ; 1$, we obtain

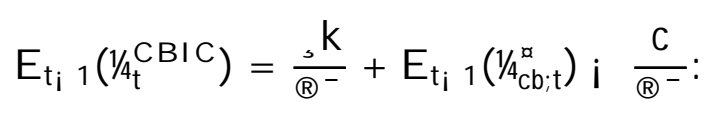

Supposing the central bank to achieve its own desired in ation rate $1 / \frac{\mathrm{a}}{\mathrm{tb}, \mathrm{t}} ;$; the rational expectation about the $i^{\circ}$ ation rate $1 / \mathrm{BIC}^{\mathrm{BC}}$ by the society at time $\mathrm{t} \mathrm{i} 1$ is then

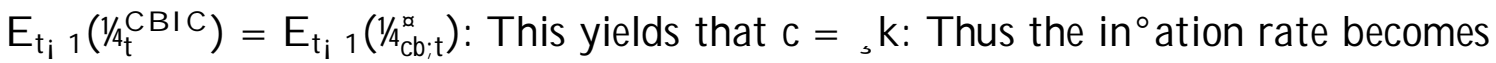

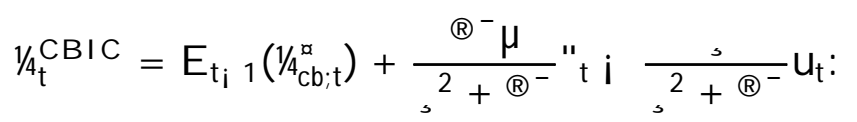

The output under the constant $i^{\circ}{ }^{\circ}$ ation contract regime is unchanged, i.e., it is the same as in (8). By comparing (7) and (15), we observe that the in ation bias $\frac{k}{\mathbb{B}^{-}}$has been completely eliminated, but the political uncertainty is untouched. The average loss function under $\mathrm{a} \mathrm{T}_{\mathbf{i}}$ term central banker evaluated at time $\mathrm{t}_{\mathbf{i}} 1$ can then be expressed as

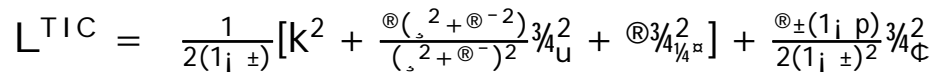

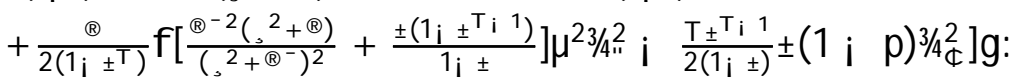

The ${ }^{-}$rst order condition for the parameter ${ }^{-}$is

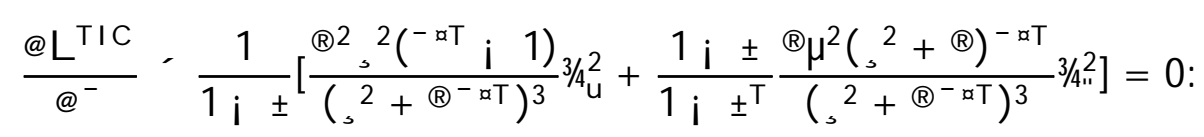

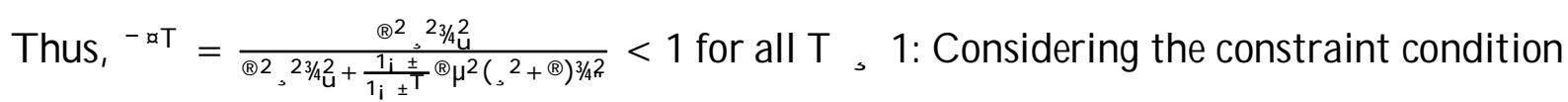
that $^{-{ }^{-} \mathrm{T}}, 1$; the optimal parameter ${ }^{-}$is al ways 1 for all $\mathrm{T}, 1:$ This indicates that a more conservative central bank is not an optimal choice under the constant in ${ }^{\circ}$ ation contract regime. Furthermore, taking this fact and condition (10) into account, the optimal term length $T$ is always 1 :

The constant in ${ }^{\circ}$ ation target is however not plausible, because the median voter (the government) would assign the in ${ }^{\circ}$ ation contract according to the realization of the shock ": In other words, since the in ${ }^{\circ}$ ation contract is assigned after the election, it could be contingent on the state of the shock "; namely, $c=a_{i} b^{\prime \prime} t$ : The rational expectation of the in $^{\circ}$ ation rate should be based on this information. As a result, the constant part $a$ is the same as that under the constant contract regime, that is, $a=, k$ : Taking the expectation on both sides of the ${ }^{-}$rst order conditions (14), based on the information after the election and considering the fact that $a=, k$; we have

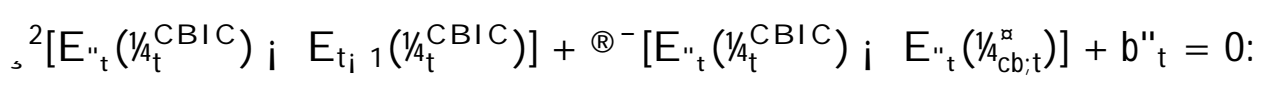




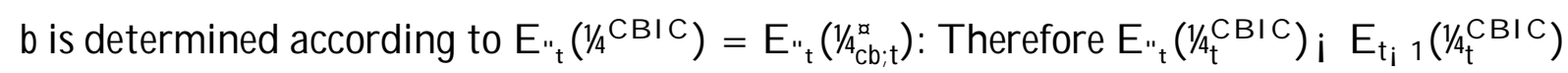
is $\mu^{\prime \prime} \mathrm{t}$ : Thus the optimal value of parameter $b$ is,${ }^{2} \mu$ : The corresponding in ation and output are then

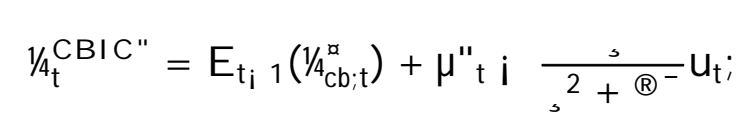

and

$$
y_{t}^{C B I C "}=y^{N}+, \mu^{\prime \prime}{ }_{t}+\frac{{ }^{-}}{{ }^{2}+\circledR^{-}} u_{t}
$$

respectively. By comparing (17) and (18) with (7) and (8), respectively, we notice that the average $i^{\circ}$ ation bias $\frac{\frac{k}{6}}{\mathbb{B}^{-}}$has been completely eliminated under both the constant and the state-contingent $\mathrm{in}^{\circ}$ ation target regimes, but the political uncertainties in both the $\mathrm{in}^{\circ}$ ation rate and the output have been increased under the state-contingent $i^{\circ}{ }^{\circ}$ ation contract regime. The economic intuition is fairly simple, that is the median voter (the government) tries to increase its partisan in ${ }^{\circ}$ uence on monetary policy. This in ${ }^{\circ}$ uence works via the central bank's desired in ${ }^{\circ}$ ation rate. The statecontingent in ${ }^{\circ}$ ation contract enables the central bank to carry out the monetary policy so as to approach its desired level even more closely.

The present valued average loss for a multi-term central banker evaluated at time $t_{i} 1$ becomes

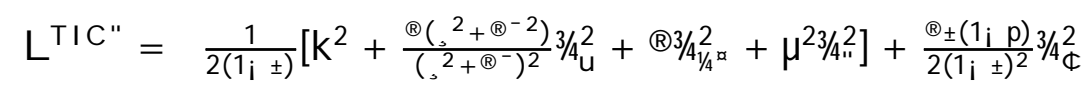

$$
\begin{aligned}
& +\frac{\Theta}{2\left(1_{i} \pm\right)}\left[\frac{2}{\Theta} \mu^{23 / 4} ; \quad \frac{T \pm}{1_{i} \pm}(1 ; \quad p)^{3 / 4}\right] \text { : }
\end{aligned}
$$

The ${ }^{-}$rst order condition for minimizing the loss function (19) with respect to the parameter ${ }^{-}$is

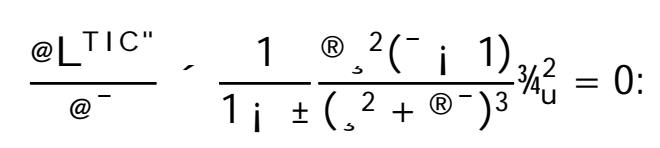

Thus the optimal conservativeness is 1 : The di Berential of $L^{\text {TIC" }}$ with respect to the term-length $T$ is

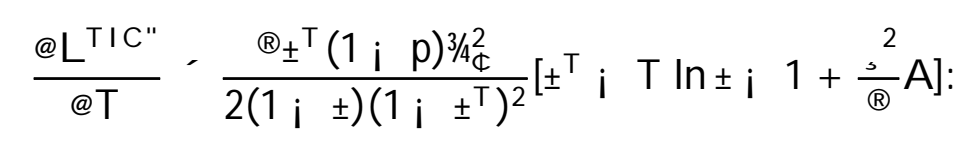

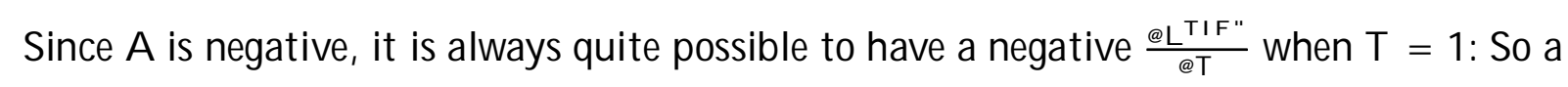
further increase in the term length would lead to a decrease in the expected loss. In other words, an optimal multi-term central banker is possible. The economic intuition for a multi-term central banker under the state contingent in ${ }^{\circ}$ ation contract is straightforward. The contingent in ${ }^{\circ}$ ation contract eliminates the in ${ }^{\circ}$ ation bias but at the cost of higher 
political risk. This can be interpreted as adding a new cost to the social loss function, if we take the constant in ${ }^{\circ}$ ation contract regime as a benchmark of which the optimal term length is one period. This cost can be partly removed by a multi-term central banker.

\section{2 $\ln ^{\circ}$ ation Target}

We now suppose that the society has adopted an in ${ }^{\circ}$ ation target regime. Thus, the parameter of conservativeness ${ }^{-}$and the term length $\mathrm{T}$ are delegated together with an in ${ }^{\circ}$ ation target $1 / 4 \mathrm{~F}$; according to the median voter's preference. We also suppose that the implicit output target is $y^{N}+k$ for all sectors. The new objective function of the central bank under the in ${ }^{\circ}$ ation target regime is therefore

$$
L_{t}^{C B I F}=\frac{1}{2}\left[\left(y_{t}^{C B} i y^{N} ; k\right)^{2}+{ }^{\circledR}\left(1 / t^{C B} ; 1 / t^{F}\right)^{2}\right]:
$$

The ${ }^{-}$rst order condition for minimizing the new loss function (20) is

$$
, f,\left[1 / \mathbb{t}^{B I F} ; E_{t_{i} 1}\left(1 / t^{B} B \mid F\right)\right]+u_{t} i k g+\circledast^{-}\left(1 / \mathbb{C}^{B} \mid F ; 1 / t^{F}\right)=0:
$$

We ${ }^{-}$rst consider the constant in ${ }^{\circ}$ ation target $1 / \mathrm{f}^{\mathrm{F}}=\mathrm{h}$ : By taking the expectation on both sides of (21) and assuming that the in ${ }^{\circ}$ ation would ful ${ }^{-} \|$the condition $E_{t_{i}}\left(1 / \mathbb{L}^{\mathbb{B} I F}\right)=$ $E_{t_{1} 1}\left(1 / \mathrm{t}_{\mathrm{t}, \mathrm{t}}\right)$, the in ${ }^{\circ}$ ation target should be valued as

$$
h\left(=1 / \mathrm{t}^{\mathrm{F}}\right)=\mathrm{E}_{\mathrm{t}_{\mathrm{i}} 1}\left(1 / \mathrm{s} \mathrm{t} \mathrm{b}_{\mathrm{t}}\right) \mathrm{i}, \frac{\mathrm{k}}{\mathbb{R}^{-}}:
$$

Accordingly, the in ${ }^{\circ}$ ation and output become

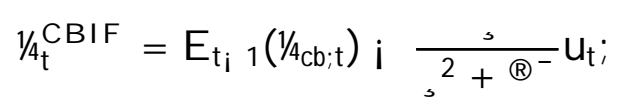

and

$$
y_{t}^{C B I F}=y^{N}+\frac{\mathbb{B}^{-}}{2^{2}+\mathbb{B}^{-}} u_{t} ;
$$

respectively. Therefore, both the in ${ }^{\circ}$ ation bias and the political uncertainty have been completely eliminated from the economy. This leads to a better situation than that under the in ${ }^{\circ}$ ation contract regime.

The optimal conservativeness and the term length can be determined by minimizing the average expected loss function with a $\mathrm{T}$-term central banker:

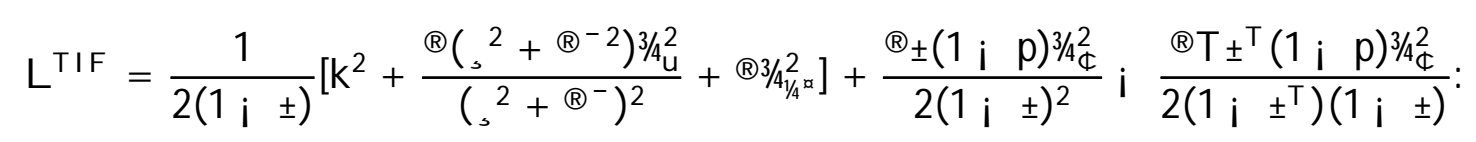


As a result, we have,

$$
-x=1 \text { and } T^{x}=1:
$$

$\mathrm{T}^{\infty}=1$ can be identi ${ }^{-}$ed as the following:

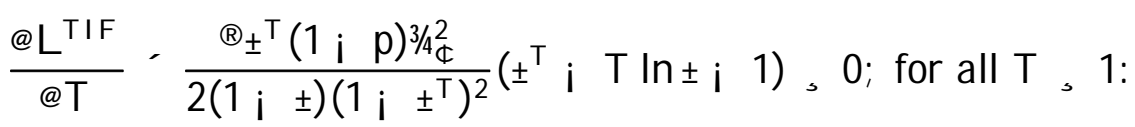

Like the constant in ${ }^{\circ}$ ation contract regime, the constant in ${ }^{\circ}$ ation target could be replaced by a state-contingent in ${ }^{\circ}$ ation target, that is, $1 / 4 \mathrm{~F}=\mathrm{h}+\mathrm{j}$ " $t$; where $\mathrm{h}$ and $\mathrm{j}$ are constants. This is because the constant in ${ }^{\circ}$ ation target is less plausible even though the society bene ${ }^{-}$ts from it. The state-contingent in ${ }^{\circ}$ ation target is imposed by the median voter (the government), which would at least preserve its in ${ }^{\circ}$ uence on the monetary policy decision. Furthermore, the central bank is also willing to allow an in ation target to be contingent on the state of the shock " $t$; since its own desired in ${ }^{\circ}$ ation rate (4) is state-contingent.

Rewriting the ${ }^{-}$rst order condition, we have

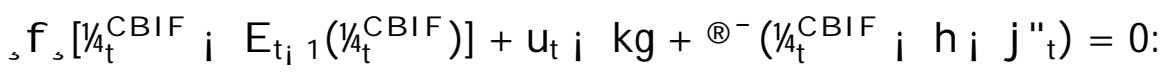

$\mathrm{h}$ and $\mathrm{j}$ can be optimally determined according to (25) and the median voter's preference,

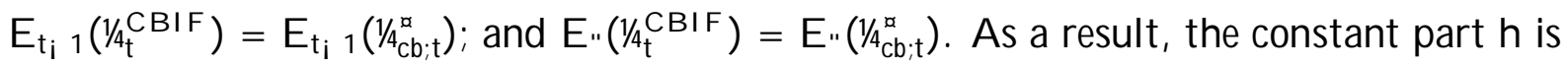
the same as that in (22), and

$$
j=\left(\frac{2}{\circledR)^{-}}+1\right) \mu:
$$

By substituting (22) and (26) into the ${ }^{-}$rst order condition (25), we obtain the in ${ }^{\circ}$ ation rate and the output under the state-contingent in ${ }^{\circ}$ ation target regime. We have noticed that both these are the same as under the state-contingent in $^{\circ}$ ation contract regime, that is, $1 / \mathbb{C}^{\mathrm{B} I F} "=1 / \mathbb{C B I C}^{\prime}$ and $\mathrm{y}_{\mathrm{t}}^{\mathrm{CBIF}}=\mathrm{y}_{\mathrm{t}}^{\mathrm{CBIC}}$ ": Therefore the equilibria under two statecontingent regimes are equivalent. Thus, the optimal parameter of conservativeness under the state-contingent $\mathrm{in}^{\circ}$ ation target regime is always 1 and the optimal term length could be more than one period.

Our main conclusions are summarized in Table 1. Even though the parameter can be determined independently of the term length $\mathrm{T}$; it is possible to have an optimal multi-term central banker under both state-contingent regimes.

Our discussion in this note focuses on the optimal term length. Even if the permanent shifts in long-run median voter's preferences and the optimal term length are disregarded, 
our results are still helpful in understanding the in ${ }^{\circ}$ ation target regime. The constant in ${ }^{\circ}$ ation target can improve the economy to the best possible situation, but it is less plausible. In other words, it reallocates the credibility problem rather than solving it. On the other hand, it is plausible to have a state-contingent in ${ }^{\circ}$ ation target that allows the in ${ }^{\circ}$ ation surprise policy to exert its e eect to the maximum extent. Thus, there could be a trade- $0 \AA$ between the in ${ }^{\circ}$ ation bias and the politically induced variability. This is consistent with the ${ }^{-}$nding in Lin (1997).

\section{R eferences}

[1] A lesina, A lberto and Gatti, R oberta. \Independent Central Banks: Low In ${ }^{\circ}$ ation at no Cost?" American E conomic Review, May 1995, 85, 196-200.

[2] Lin, Xiang. IOptimal In ${ }^{\circ}$ ation Targets, $I n^{\circ}$ ation Contracts and Political Cycles." Unpublished Manuscript, Stockholm University, 1997.

[3] Persson, Torsten and Tabellini, Guido. IDesigning Institutions for Monetary Stability." Carnegie-Rochester Conference Series on Public Policy, December 1993, 39, 55-83.

[4] R ogo $®$, K enneth. \The Optimal Degree of Commitment to an Intermediate Monetary Target." Quarterly J ournal of E conomics, November 1985, 100, 1169-1190.

[5] Svensson, Lars. 1 Optimal In ${ }^{\circ}$ ation Targets, 'Conservative' Central Banks, and Linear In ation Contracts." American E conomic Review, March 1997, 87, 98-114.

[6] Waller, Christopher and Walsh, Carl. ICentral Bank Independence, Economic Behavior, and Optimal Term Lengths." American E conomic Review, December 1996, 86, 1139-1153.

[7] Walsh, Carl. IO ptimal Contracts for Central Bankers." American Economic Review, March 1995, 85, 150-167. 
Table 1

\begin{tabular}{|c|c|c|}
\hline & Optimal Conservativeness & Optimal Term Length \\
\hline Constant $\operatorname{In}^{\circ}$ ation Contract & $-=1^{\infty}$ & $\mathrm{T}=1$ \\
\hline state-contingent $\mathrm{In}^{\circ}$ ation Contract & $-=1$ & $\mathrm{~T}, 1$ \\
\hline Constant $I \mathrm{n}^{\circ}$ ation Target & $-1^{\infty}$ & $\mathrm{T}=1$ \\
\hline state-contingent $\mathrm{In}^{\circ}$ ation Target & $-=1$ & $\mathrm{~T}, 1$ \\
\hline
\end{tabular}

${ }^{\infty}$ obtained by the constraint condition ${ }^{-}, 1$ : 
Figure 1

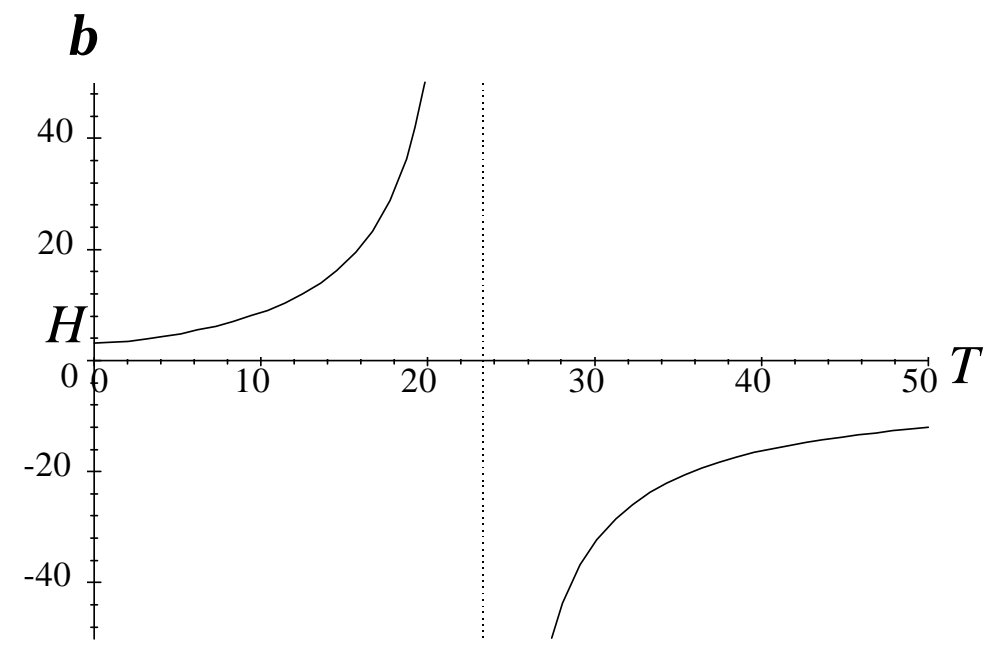

The dot line represents the asymptotic line.

Values of parameters:

$囚=2: 5 ;,=3 ; \pm=0: 7 ; \mu=0: 9 ; p=: 9 ; k^{2}=2: 5 ; 3 / 2=: 5 ; 3 / 4=30 ; 3 / 4=: 9:$ 
F igure 2

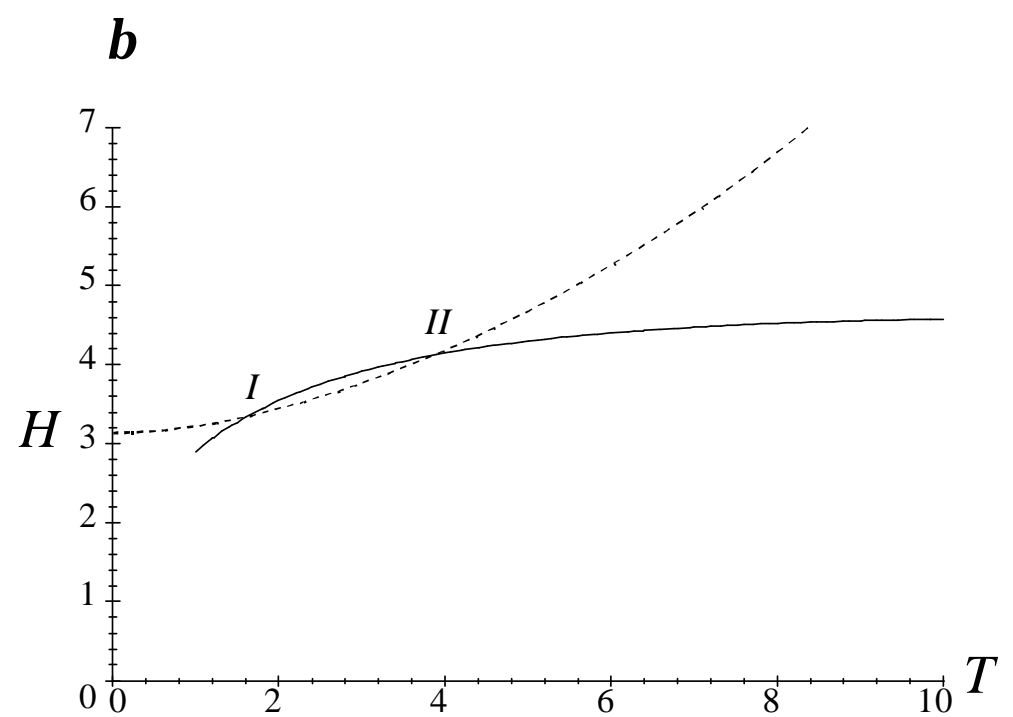

Values of parameters are the same as those in Figure 1. 